\title{
Odd central moments of unimodal distributions
}

\author{
Claude Bélisle \\ Department of Statistics, University of Michigan, Ann Arbor, MI 48109-1027, USA
}

Received October 1989

Revised March 1990

\begin{abstract}
We present a simple geometric condition under which all existing odd central moments of a unimodal distribution are non-negative. The criterion applies to both the absolutely continuous case and the lattice case. In the lattice case, the result proves and generalizes a conjecture of Frame, Gilliland and Hsing. In the absolutely continuous case, the result provides a new proof of results of Hannan and Pitman, Runnenburg, and MacGillivray. The main idea is a new decomposition result for unimodal distributions.
\end{abstract}

AMS 1980 Subject Classification: Primary 60E15.

Keywords: Unimodal distribution, symmetric distribution, skewness, central moments.

\section{Introduction}

Frame and Gilliland $(1985$, p. 38$)$ conjectured that if $X$ is a non-negative integer valued random variable with non-increasing probability mass function and with finite mean $\mu$ then all its odd central moments are non-negative:

$$
\boldsymbol{E}\left[(X-\mu)^{2 k+1}\right] \geqslant 0, \quad k=1,2,3, \ldots
$$

The conjecture has been established for a variety of special cases (Gilliland and Hsing, 1988) but the general problem has remained unsolved (see also Gill and Gilliland, 1989, p. 66).

In the absolutely continuous case, the above odd central moment inequality does hold. In fact, inspired by a conjecture of Birnbaum (1950) on the sign of the skewness of truncated normal distributions, Hannan and Pitman (1965) have shown that if $X$ has a symmetric unimodal probability density function and if $\boldsymbol{E}[|\mathrm{X}|]<\infty$ then for every $c$ with $\boldsymbol{P}[X>c]>0$, the conditional distribution of $X$ given that $X>c$ has non-negative odd central moments of all orders. Their proof does not seem to work in the lattice case. More generally, Runnenburg (1978, Remark (a)) and MacGillivray (1981, Section 2) have shown that if $X$ has a non-symmetric unimodal probability density function $f$ such that $(f(\mu+x)-f(\mu-x) ; x \geqslant 0)$ changes sign at most once and does so from negative to positive, then all existing odd central moments are positive. Again, their proof does not seem to work in the lattice case. (See Remark 1 below.)

The purpose of this paper is to present a simple geometric condition, condition (1) below, under which all existing odd central moments of a unimodal distribution are non-negative. Our criterion applies to both the lattice case and the absolutely continuous case. In the lattice case, our result generalizes the Frame-Gilliland-Hsing conjecture. In the continuous case it provides a different proof of the Runnen- 
burg-MacGillivray result. In Section 2 we introduce some notation and we state the main result (Theorem 2.1). The main idea of our approach is the decomposition result presented in Section 3. We show that if a unimodal distribution satisfies condition (1) below, then it can be written as a mixture of positively skewed bi-uniform distributions (see Definition 3.4 below) all having the same mean. In Section 4 we show that the positively skewed bi-uniform distributions have positive odd central moments. The main result then follows from the decomposition result.

Remark 1. If $X$ is integer valued and if $Y=X+U$ where $U$ is uniformly distributed over the interval $\left(-\frac{1}{2}, \frac{1}{2}\right)$ and is independent of $X$, then $X$ and $Y$ have the same mean and the same third central moment. However their higher odd central moments need not be equal and therefore positively of the odd central moments of $X$ is not an immediate consequence of the positivity of the odd central moments of $Y$.

\section{Statement of the result}

Let $F$ be a probability measure on the real line $\mathbb{R}$. For $d>0$ let $L_{d}=\{k d ; k \in \mathbb{Z}\}$, the lattice with span $d$, and let $m_{d}$ be $d$ times counting measure on $L_{d}$. The real line $\mathbb{R}$ will also be denoted $L_{0}$, the Borel $\sigma$-field on $\mathbb{R}$ will be denoted $\mathscr{B}$, and the Lebesgue measure on $\mathbb{R}$ will be denoted $m_{0}$. Now assume that $F$ is unimodal, i.e. assume that for some $d \geqslant 0, F$ is absolutely continuous with respect to $m_{d}$ and some version of its density function with respect to $m_{d}$ is non-decreasing on $\left(-\infty, x_{0}\right] \cap L_{d}$ and non-increasing on $\left[x_{0}, \infty\right) \cap L_{d}$, for some $x_{0} \in \mathbb{R}$. In the lattice case $(d>0)$ let $f$ be the version of the density of $F$ with respect to $m_{d}$ given by

$$
f(x)=F(\{x\}) / d \quad \forall x \in \mathbb{R},
$$

and in the continuous case $(d=0)$ let $f$ be the right continuous version of the density of $F$ with respect to $m_{0}$. With this choice of $f$, let

$$
y_{\max }=\sup _{x \in \mathbb{R}} f(x)
$$

and for $0<y<y_{\max }$, let

$$
h_{F}(y)=\frac{\inf \{x \in \mathbb{R}: f(x)>y\}+\sup \{x \in \mathbb{R}: f(x)>y\}}{2} .
$$

Thus $h_{F}(y)$ is the middle of the horizontal cross section of the graph of $f$ at $y$. (See Figure 1 and Figure 2 below.) Recall that $F$ is said to be symmetric if there is an $x$ such that $F(x-B)=F(x+B)$ for every Borel set $B$. Otherwise it is said to be non-symmetric. Observe that if $F$ is symmetric and has mean $\mu_{F}$ then $h_{F}(y)=\mu_{F}$ for all $y \in\left(0, y_{\max }\right)$ where if $F$ is non-symmetric and has finite mean $\mu_{F}$ then the scts $\left\{y \in\left(0, y_{\max }\right): h_{F}(y)<\mu_{F}\right\}$ and $\left\{y \in\left(0, y_{\max }\right): h_{F}(y)>\mu_{F}\right\}$ are both non-empty.

Theorem 2.1. Let $F$ be a unimodal probability measure with finite mean $\mu_{F}$. Suppose that $F$ is non-symmetric and that

$$
\inf \left\{y \in\left(0, y_{\max }\right): h_{F}(y)<\mu_{F}\right\} \geqslant \sup \left\{y \in\left(0, y_{\max }\right): h_{F}(y)>\mu_{F}\right\} .
$$

Then, for $k=1,2,3, \ldots$,

$$
\int_{\mathbb{R}}\left(x-\mu_{F}\right)^{2 k+1} F(\mathrm{~d} x)>0
$$

whenever the left hand side is well defined. 
Observe that if $F$ is non-symmetric and $h_{F}$ is non-increasing then condition (1) is satisfied. And if $F$ is symmetric then all existing odd central moments are null. Thus the following corollary is an immediate consequence of Theorem 2.1.

Corollary 2.2. Let $G$ be a symmetric unimodal probability measure with mean $\mu_{G}$. Let $c>-\infty$ be such that $G((c, \infty))>0$, let $F$ be the probability measure defined by

$$
F(B)=G(B \cap(c, \infty)) / G((c, \infty)) \quad \forall B \in \mathscr{B}
$$

and let $\mu_{F}$ denote its mean. Then, for $k=1,2,3, \ldots$,

$$
\int_{\mathbb{R}}\left(x-\mu_{F}\right)^{2 k+1} F(\mathrm{~d} x) \geqslant 0 .
$$

Furthermore, equality holds if and only if $F$ is symmetric.

Proof. Let $G$ and $c$ be as in the statement of the corollary. If $G(c, \infty)=1$, then $F$ is symmetric (since it is equal to $G$ itself) and its odd central moments exist (since $F$ then has bounded support). Thus $\int_{\mathbf{R}}\left(x-\mu_{F}\right)^{2 k+1} F(\mathrm{~d} x)=0$ for $k=1,2,3, \ldots$ If $G(c, \infty)<1$, then clearly $F$ is non-symmetric and $h_{F}$ is non-increasing. Furthermore $F$ has finite mean (since $G$ does) and all its odd central moments are well defined (possibly equal to $+\infty$ ) since the support of $F$ is bounded below. Thus Theorem 2.1 yields $\int_{\mathbb{R}}\left(x-\mu_{F}\right)^{2 k+1} F(\mathrm{~d} x)>0$ for $k=1,2,3, \ldots$

Our proof of Theorem 2.1 is based on the following elementary application of Fubini's theorem which we state as a lemma for easy reference. Roughly speaking, it says that if a probability measure $F$ is a mixture (see Definition 3.5 below) of probability measures all having the same mean and all having positive $(2 k+1)$ st central moment, then $F$ itself has positive $(2 k+1)$ st central moment.

Lemma 2.3. Let $F$ be a probability measure with finite mean $\mu_{F}$. Suppose that

$$
F(B)=\int_{Y} F_{y}(B) Q(\mathrm{~d} y) \quad \forall B \in \mathscr{B}
$$

where $(Y, \mathscr{Y}, Q)$ is a probability space and where $\left(F_{y}(B) ; y \in Y, B \in \mathscr{B}\right)$ satisfies

(i) for all $B \in \mathscr{B}, F(B)$ is $\mathscr{S}$-measurable,

(ii) for every $y, F_{y}(\cdot)$ is a probability measure on $\mathbb{R}$ with mean $\mu_{F_{y}}$,

(iii) for every $y, \mu_{F_{v}}=\mu_{F}$.

Let $k$ be a positive integer. Assume that for every $y, \int_{\mathbf{R}}\left(x-\mu_{F}\right)^{2 k+1} F_{y}(\mathrm{~d} x)$ is well defined. Assume also that $\int_{\mathbb{R}}\left(x-\mu_{F}\right)^{2 k+1} F(\mathrm{~d} x)$ is well defined. Then:

(a) If

$$
Q\left(\left\{y \in Y: \int_{\mathbb{R}}\left(x-\mu_{F}\right)^{2 k+1} F_{y}(\mathrm{~d} x) \geqslant 0\right\}\right)=1,
$$

then

$$
\int_{\mathbf{R}}\left(x-\mu_{F}\right)^{2 k+1} F(\mathrm{~d} x) \geqslant 0 .
$$


(b) If

$$
Q\left(\left\{y \in Y: \int_{\mathbb{R}}\left(x-\mu_{F}\right)^{2 k+1} F_{y}(\mathrm{~d} x) \geqslant 0\right\}\right)=1
$$

and

$$
Q\left(\left\{y \in Y: \int_{\mathbf{R}}\left(x-\mu_{F}\right)^{2 k+1} F_{y}(\mathrm{~d} x)>0\right\}\right)>0
$$

then

$$
\int_{\mathbb{R}}\left(x-\mu_{F}\right)^{2 k+1} F(\mathrm{~d} x)>0 .
$$

Note that in the statement of this lemma, one can (in view of conditions (i), (ii) and (iii)) think of $\left(F_{y}(B) ; y \in Y, B \in \mathscr{B}\right)$ as a Markov kernel (of transition probabilities from $(Y, \mathscr{Y})$ to $(\mathbb{R}$, $\mathscr{B})$ ) with constant mean.

Remark 2. In the absolutely continuous case, condition (1) of Theorem 2.1 is equivalent to the condition of Runnenburg and MacGillivray stated in Section 1 above.

Remark 3. In the discrete case we are requiring that $F$ be lattice unimodal, i.e. we are requiring that there exist a $d>0$ and an $x_{0} \in \mathbb{R}$ such that $F\left(L_{d}\right)=1$ and $F(x)$ is non-decreasing on $\left(-\infty, x_{0}\right] \cap L_{d}$ and non-increasing on $\left[x_{0}, \infty\right) \cap L_{d}$. The following example shows that if $F$ is merely 'unimodal on its supports', then the conclusion of Theorem 2.1 need not hold, even if condition (1) is satisfied. Put mass 0.42 at 0 , mass 0.38 at 2 , and mass 0.2 to 3 . Then $F$ is 'unimodal on its support' and condition (1) is satisfied, but the third central moment of $F$ is negative.

\section{Proof of 'Theorem 2.1}

First we consider the case where the set $\left\{y \in\left(0, y_{\max }\right): h_{F}(y)=\mu_{F}\right\}$ has Lebesgue measure 0 . In this case equality holds in (1). Let

$$
y_{*}=\inf \left\{y \in\left(0, y_{\max }\right): h_{F}(y)<\mu_{F}\right\}=\sup \left\{y \in\left(0, y_{\max }\right): h_{F}(y)>\mu_{F}\right\}
$$

and for $y \in\left(0, y_{*}\right)$ let $\beta(y)$ be the unique number in $\left(y_{*}, y_{\max }\right)$ such that

$$
\int_{y}^{\beta(y)} \int_{\left[h^{(}(u), h_{+}(u)\right]}\left(x-\mu_{F}\right) m_{d}(\mathrm{~d} x) \mathrm{d} u=0
$$

where $h_{+}(u)=\sup \left\{x \in L_{d}: f(x)>u\right\}$ and $h_{-}(u)=\inf \left\{x \in L_{d}: f(x)>u\right\}$. For the case $d=0$, the geometric interpretation of $\beta(y)$ is that in Figure 1, the $x$-coordinate of the center of gravity of the shaded region is $\mu_{F}$. A similar geometric interpretation holds for the case $d>0$.

\section{Proposition 3.1.}

(a) $\beta$ is strictly decreasing on $\left(0, y_{*}\right)$.

(b) $\beta$ is absolutely continuous on $\left(0, y_{*}\right)$.

(c) For almost every $y$ in $\left(0, y_{*}\right)$, the derivative $\beta^{\prime}(y)$ exists, is negative, and satisfies

$$
\beta^{\prime}(y)=\frac{\left(h_{F}(y)-\mu_{F}\right) m_{d}\left(\left[h_{-}(y), h_{+}(y)\right]\right)}{\left(h_{F}(\beta(y))-\mu_{F}\right) m_{d}\left(\left[h_{-}(\beta(y)), h_{+}(\beta(y))\right]\right)} .
$$




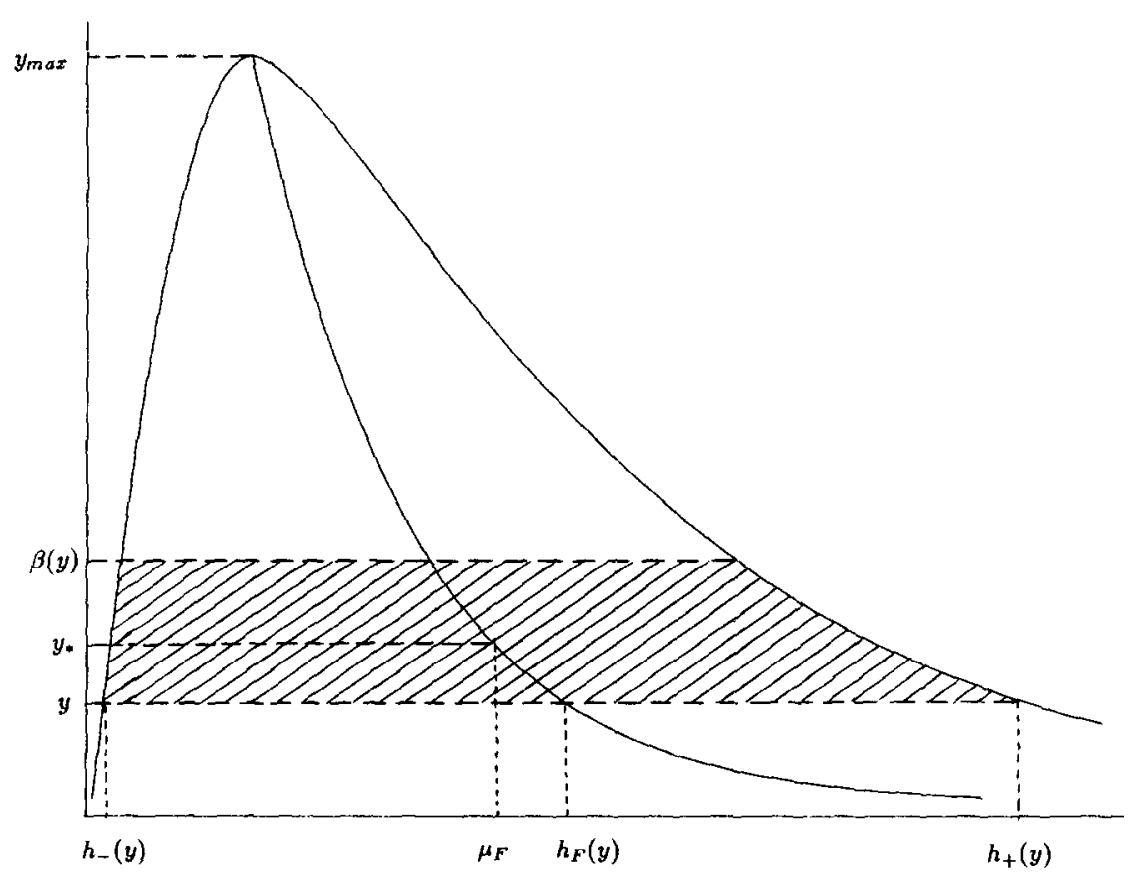

Fig. 1.

Proof. Observe that $h_{-}(u)$ and $h_{+}(u)$, and hence $\int_{\left[h_{-}(u), h_{+}(u)\right]}\left(x-\mu_{F}\right) m_{d}(\mathrm{~d} x)$, are continuous in $u$ almost everywhere in $\left(0, y_{\max }\right)$. Parts (a) and (b) follow at once. Now fix $y \in\left(0, y_{*}\right)$. For $\Delta y \neq 0$ such that $y+\Delta y+\left(0, y_{*}\right)$, equation (2) yields

$$
\int_{\beta(y)}^{\beta(y+\Delta y)} \int_{\left[h_{-}(u), h_{+}(u)\right]}\left(x-\mu_{F}\right) m_{d}(\mathrm{~d} x) \mathrm{d} u-\int_{y}^{y+\Delta y} \int_{\left[h_{-}(u), h_{+}(u)\right]}\left(x-\mu_{F}\right) m_{d}(\mathrm{~d} x) \mathrm{d} u=0 .
$$

Thus by continuity we obtain, for almost every $y \in\left(0, y_{*}\right)$,

$$
\begin{gathered}
\left(\int_{\left[h_{-}(\beta(y)) \cdot h_{+}(\beta(y))\right]}\left(x-\mu_{F}\right) m_{d}(\mathrm{~d} x)+\mathrm{o}(1)\right)(\beta(y+\Delta y)-\beta(y)) \\
-\left(\int_{\left[h_{-}(y), h_{+}(y)\right]}\left(x-\mu_{F}\right) m_{d}(\mathrm{~d} x)+\mathrm{o}(1)\right) \Delta y=0 \quad \text { as } \Delta y \rightarrow 0 .
\end{gathered}
$$

Note that for every $y \in\left(0, y_{*}\right)$,

$$
\begin{aligned}
& \int_{\left[h_{-}(y), h_{+}(y)\right]}\left(x-\mu_{F}\right) m_{d}(\mathrm{~d} x)=\left(h_{F}(y)-\mu_{F}\right)\left(m_{d}\left(\left[h_{-}(y), h_{+}(y)\right]\right)\right)>0, \\
& \int_{\left[h_{-}(\beta(y)), h_{+}(\beta(y))\right]}\left(x-\mu_{F}\right) m_{d}(\mathrm{~d} x)=\left(h_{F}(\beta(y))-\mu_{F}\right)\left(m_{d}\left(\left[h_{-}(\beta(y)), h_{+}(\beta(y))\right]\right)\right)<0 .
\end{aligned}
$$

Thus solving for $(\beta(y+\Delta Y)-\beta(y)) / \Delta y$ and letting $\Delta y \rightarrow 0$ yields part (c).

If $y$ is such that $\beta^{\prime}(y)$ exists, is negative, and satisfies (3), then let

$$
F_{y}(B)=\frac{m_{d}\left(\left[h_{-}(y), h_{+}(y)\right] \cap B\right)-\beta^{\prime}(y) m_{d}\left(\left[h_{-}(\beta(y)), h_{+}(\beta(y))\right] \cap B\right)}{m_{d}\left(\left[h_{-}(y), h_{+}(y)\right]\right)-\beta^{\prime}(y) m_{d}\left(\left[h_{-}(\beta(y)), h_{+}(\beta(y))\right]\right)} \quad \forall B \in \mathscr{B},
$$


and

$$
q(y)=m_{d}\left(\left[h_{-}(y), h_{+}(y)\right]\right)-\beta^{\prime}(y) m_{d}\left(\left[h_{-}(\beta(y)), h_{+}(\beta(y))\right]\right) .
$$

Otherwise, let $F_{y}(\cdot)$ be point mass at $\mu_{F}$ and let $q(y)=0$. Finally, let

$$
Q(B)=\int_{\left(0, y_{*}\right) \cap B} q(y) \mathrm{d} y \quad \forall B \in \mathscr{B}_{\left(0, y_{*}\right)}
$$

where $\mathscr{B}_{\left(0, y_{*}\right)}$ denotes the Borel $\sigma$-field on $\left(0, y_{*}\right)$.

\section{Proposition 3.2.}

(a) $\forall B \in \mathscr{B}, F$. $(B)$ is measurable.

(b) For all $y \in\left(0, y_{*}\right), F_{y}(\cdot)$ is a probability measure on $L_{d}$.

(c) For all $y \in\left(0, y_{*}\right), F_{y}(\cdot)$ has mean $\mu_{F}$.

(d) $Q$ is a probability measure on $\left(0, y_{*}\right)$.

(e) $\forall B \in \mathscr{B}, F(B)=\int_{\left(0, y_{*}\right)} F_{y}(B) Q(\mathrm{~d} y)$.

Proof. Parts (a) and (b) are consequences of the definitions and part (c) follows from (3). For $B \in \mathscr{B}$,

$$
\begin{aligned}
F(B) & =\int_{B} f(x) m_{d}(\mathrm{~d} x) \\
& =\int_{\mathbf{R}} \int_{0}^{f(x)} 1_{B}(x) \mathrm{d} y m_{d}(\mathrm{~d} x) \\
& =\int_{0}^{y_{\max }} \int_{\left[h_{-}(y), h_{+}(y)\right]} 1_{B}(x) m_{d}(\mathrm{~d} x) \mathrm{d} y \\
& =\int_{0}^{y_{*}} \int_{\left[h_{-}(y), h_{+}(y)\right]} 1_{B}(x) m_{d}(\mathrm{~d} x) \mathrm{d} y+\int_{y_{*}}^{y_{\max }} \int_{\left[h_{-}(y), h_{+}(y)\right]} 1_{B}(x) m_{d}(\mathrm{~d} x) \mathrm{d} y \\
& =\int_{0}^{y_{*}} \int_{\left[h_{-}(y), h_{+}(y)\right]} 1_{B}(x) m_{d}(\mathrm{~d} x) \mathrm{d} y-\int_{0}^{y_{*}} \beta^{\prime}(y) \int_{\left[h_{-}(\beta(y)), h_{+}(\beta(y))\right]} 1_{B}(x) m_{d}(\mathrm{~d} x) \mathrm{d} y \\
& =\int_{0}^{y_{*}}\left\{m_{d}\left(\left[h_{-}(y), h_{+}(y)\right] \cap B\right)-\beta^{\prime}(y) m_{d}\left(\left[h_{-}(\beta(y)), h_{+}(\beta(y))\right] \cap B\right)\right\} \mathrm{d} y \\
& =\int_{0}^{y_{*}} F_{y}(B) Q(\mathrm{~d} y) .
\end{aligned}
$$

This proves part (d) and (e).

Proposition 3.3. For every integer $k \geqslant 1$,

$$
Q\left(\left\{y \in\left(0, y_{*}\right): \int_{\mathbb{R}}\left(x-\mu_{F}\right)^{2 k+1} F_{y}(\mathrm{~d} x)>0\right\}\right)=1 .
$$

Proof. For $u, v \in L_{d}$, with $u \leqslant v$, let $U_{u, v}^{(d)}$ denote the uniform probability measure on $[u, v] \cap L_{d}$. For almost every $y$ in $\left(0, y_{*}\right)$, the probability measure $F_{y}$ defined in (4) is of the form $F_{y}=p U_{s, t}^{(d)}$ $+(1-p) U_{r, u}^{(d)}$ with $0<p<1$ and $r, s, t, u \in L_{d}$ such that $r \leqslant s \leqslant t<u, \frac{1}{2}(r+u)>\frac{1}{2}(s+t)$, and $\frac{1}{2} p(s+t)$ $+\frac{1}{2}(1-p)(r+u)=\mu_{F}$. Theorem 4.5 , below, asserts that such probability measures have strictly positive $(2 k+1)$ st central moments for every positive integer $k$. 
The conclusion of Theorem 2.1 now follows at once from Proposition 3.2, Proposition 3.3 and Lemma 2.3. Finally, consider the case where the set $\left\{y \in\left(0, y_{\max }\right): h_{F}(y)=\mu_{F}\right\}$ has strictly positive Lebesgue measure. Let

$$
\begin{aligned}
& S=\left\{y \in\left(0, y_{\max }\right): h_{F}(y)=\mu_{F}\right\}, \quad \alpha=\int_{S} m_{d}\left(\left[h_{-}(u), h_{+}(u)\right]\right) \mathrm{d} u, \\
& F^{(1)}(B)=\frac{1}{\alpha} \int_{S} m_{d}\left(\left[h_{-}(u), h_{+}(u)\right] \cap B\right) \mathrm{d} u \quad \forall B \in \mathscr{B}, \\
& F^{(2)}(B)=\frac{1}{1-\alpha}\left\{F(B)-\int_{S} m_{d}\left(\left[h_{-}(u), h_{+}(u)\right] \cap B\right) \mathrm{d} u\right\} \quad \forall B \in \mathscr{B} .
\end{aligned}
$$

Then $F=\alpha F^{(1)}+(1-\alpha) F^{(2)}$. Observe that $0<\alpha<1$ and that $F^{(1)}$ and $F^{(2)}$ are both probability measures with mean $\mu_{F}$. By symmetry

$$
\int_{\mathbb{R}}\left(x-\mu_{F}\right)^{2 k+1} F^{(1)}(\mathrm{d} x)=0, \quad k=1,2,3, \ldots
$$

By the special case treated above,

$$
\int_{\mathbf{R}}\left(x-\mu_{F}\right)^{2 k+1} F^{(2)}(\mathrm{d} x)>0, \quad k=1,2,3, \ldots
$$

Theorem 2.1 now follows from Lemma 2.3.

Definition 3.4. A probability measure on $L_{d}$ is called a nested bi-uniform distribution (on $L_{d}$ ) if it is of the form $p U_{s, t}^{(d)}+(1-p) U_{r . u}^{(d)}$ for some $0 \leqslant p \leqslant 1$ and some $r, s, t, u \in L_{d}$ with $r \leqslant s \leqslant t \leqslant u$. It is called a positively skewed nested bi-uniform distribution (on $L_{d}$ ) if, in addition, $\frac{1}{2}(s+t) \leqslant \frac{1}{2}(r+u)$.

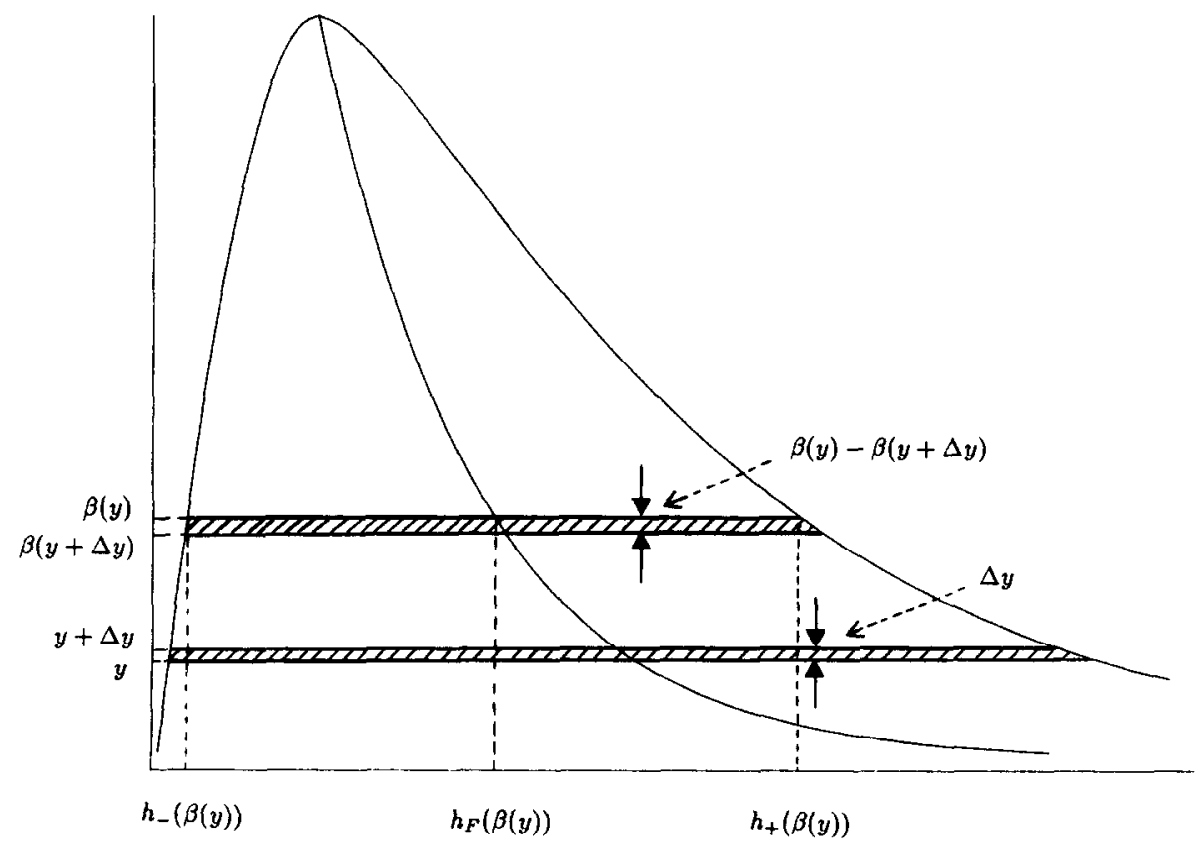

Fig. 2. 
The probability measure $F_{y}$ defined by equation (4) is a nested bi-uniform distribution on $L_{d}$. Figure 2 gives, for the case $d=0$, a geometric interpretation of $F_{y} q(y) \Delta y$ as a mixture of a 'horizontal slice' of $F$ at $y$ and a 'horizontal slice' of $F$ at $\beta(y)$ where the relative 'thickness of the slices' is such that $F_{y}$ has mean $\mu_{F}$. By 'piling up' the $F_{y} q(y) \Delta y$ 's we recover $F$. A similar geometric interpolation holds for the case $d>0$. The following definition is standard:

Definition 3.5. Let $F$ be a probability measure on $\mathbb{R}$. Let $\left(F_{y} ; y \in Y\right)$ be a family of prohability measure on $\mathbb{R}$. We say that $F$ is a mixture of the $F_{y}$ 's if there exists a $\sigma$-field $\mathscr{Y}$ on $Y$ and a probability measure $Q$ on $(Y, \mathscr{Y})$ such that for every Borel set $B$ in $\mathbb{R}$, the function $F_{y}(B)$ is $\mathscr{Y}$-measurable and $F(B)=$ $\int_{Y} F_{y}(B) Q(\mathrm{~d} y)$. The probability measure $Q$ is called the mixing measure. If the support of $Q$ is a finite set, we say that the mixture is finite. If the support of $Q$ is countable, we say that the mixture is countable.

We have proved the following decomposition result:

Theorem 3.6. Fix $d \geqslant 0$. Let $F$ be a probability measure on $L_{d}$, with finite mean. Assume that $F$ satisfies condition (1). Then $F$ is a mixture of positively skewed nested bi-uniform distributions on $L_{d}$, all having the same mean.

Remark 4. A close look at the proof of the above theorem shows that if $f$ has finite range then the mixture can be made finite and if $f$ has countable range then the mixture can be made countable. (This follows from the fact that if $f$ has finite (respectively countable) range, then the functions $h_{F}, h_{-}$, and $h_{+}$are all finite (respectively countable) linear combinations of indicator functions of disjoint intervals. The details are omitted.) In particular this always applies when $d>0$.

\section{Odd central moments of nested bi-uniform distributions}

If it exists, the mean of a probability measure $F$ will be denoted $\mu(F)$ or simply $\mu$ if there is no ambiguity. The $j$ th central moment, if it is well defined, will be denoted $\mu_{(j)}(F)$. The probability measure $F(B)=1_{B}(a), B \in \mathscr{B}$, will be called the point mass at $a$ and will he denoted $\delta_{a}$. In the following four lemmas, $F$ is a probability measure with mean $\mu$.

Lemma 4.1. Suppose that $\mu_{(3)}(F)>0$ and that the probability measure $F(B \cap(-\infty, \mu)) / F((-\infty, \mu))$, $B \in \mathscr{B}$, is a point mass. Then $\mu_{(2 k+1)}(F)>0$ for every positive integer $k$.

Proof. Under the stated assumptions, $F$ can be written as $F=p \delta_{a}+(1-p) G$ for some $a<\mu$, some $0<p<1$, and some probability measure $G$ concentrated on the interval $[\mu, \infty)$. Hence

$$
\frac{\mu_{(2 k+1)}(F)}{(\mu-a)^{2 k+1}}=-p+(1-p) \int_{(\mu, \infty)}\left(\frac{x-a}{\mu-a}-1\right)^{2 k+1} G(\mathrm{~d} x) .
$$

If $x>\mu$, then $(x-a) /(x-\mu)-1>0$ and therefore $((x-a) /(x-\mu)-1)^{2 k+1}$ is convex in $k$. Thus the above integral is convex in $k$. Thus the quantity $\mu_{(2 k+1)}(F) /(\mu-a)^{2 k+1}$ is convex in $k$. It is equal to 0 at $k=0$ and it is strictly positive at $k=1$. This proves the lemma.

Lemma 4.2. Suppose that $F$ is of the form

$$
F=\frac{p}{m+1} \sum_{i=0}^{m} \delta_{i}+\frac{1-p}{n} \sum_{i=m+1}^{m+n} \delta_{i}
$$


where $m$ is a non-negative integer, $n$ is a positive integer, and $(m+n) /(m+n+1)<p<1$. Then $\mu_{(2 k+1)}(F)>0$ for every positive integer $k$.

Proof. Induction on $m$. The cases $m=0$ and $m=1$ follow easily from Lemma 4.1. Consider the case $m>1$. One can write $F=\pi F^{(1)}+(1-\pi) F^{(2)}$ with $\pi=p /(m+1)+b+(1-p)$, with

$$
F^{(1)}=\frac{1}{\pi}\left(\frac{p}{m+1} \delta_{0}+b \delta_{m}+\frac{1-p}{n} \sum_{i=m+1}^{m+n} \delta_{i}\right)
$$

and

$$
F^{(2)}=\frac{1}{1-\pi}\left(\frac{p}{m+1} \sum_{i=1}^{m-1} \delta_{i}+\left(\frac{p}{m+1}-b\right) \delta_{m}\right)
$$

and with $h$ such that $\mu\left(F^{(1)}\right)=\mu\left(F^{(2)}\right)=\mu$. The induction hypothesis yields $\mu_{(2 k+1)}\left(F^{(2)}\right)>0$ and Lemma 4.1 yields $\mu_{(2 k+1)}\left(F^{(1)}\right)>0$. Thus Lemma 2.3 yields $\mu_{(2 k+1)}(F)>0$.

Lemma 4.3. Suppose that $F$ is of the form

$$
F=p \delta_{0}+\frac{1-p}{n} \sum_{i=1}^{n} \delta_{i}
$$

where $n$ is a positive integer and where $1 /(n+1)<p<1$. Then $\mu_{(2 k+1)}(F)>0$ for every positive integer $k$.

Proof. Induction on $n$. The case $n=1$ follows from Lemma 4.1. Consider the case $n>1$. If $2 /(n+1)<p$ $<1$, one can write $F=\pi F^{(1)}+(1-\pi) F^{(2)}$, with $\pi=(1-p) / n+b$, with

$$
F^{(1)}=\frac{1}{\pi}\left(b \delta_{0}+\frac{1-p}{n} \delta_{n}\right) \quad \text { and } \quad F^{(2)}=\frac{1}{1-\pi}\left((p-b) \delta_{0}+\frac{1-p}{n} \sum_{i=1}^{n-1} \delta_{i}\right)
$$

and with $b$ such that $\mu\left(F^{(1)}\right)=\mu\left(F^{(2)}\right)=\mu$. The induction hypothesis yields $\mu_{(2 k+1)}\left(F^{(2)}\right)>0$ and Lemma 4.1 yields $\mu_{(2 k+1)}\left(F^{(1)}\right)>0$. Thus Lemma 2.3 yields $\mu_{(2 k+1)}(F)>0$. If $1 /(n+1)<p<2 /(n+1)$, one can write $F=\pi F^{(1)}+(1-\pi) F^{(2)}$ with $\pi=p+(1-p) / n+b$ and with

$$
F^{(1)}=\frac{1}{\pi}\left(\left(p-\frac{1-p}{n}\right) \delta_{0}+b \delta_{n}\right) \quad \text { and } \quad F^{(2)}=\frac{1}{1-\pi}\left(\frac{1-p}{n} \sum_{i=0}^{n-1} \delta_{i}+\left(\frac{1-p}{n}-b\right) \delta_{n}\right)
$$

and with $b$ such that $\mu\left(F^{(1)}\right)=\mu\left(F^{(2)}\right)=\mu$. Lemma 4.1 yields $\mu_{(2 k+1)}\left(F^{(1)}\right)>0$ and Lemma 4.2 yields $\mu_{(2 k+1)}\left(F^{(2)}\right)>0$. Thus Lemma 2.3 yields $\mu_{(2 k+1)}(F)>0$. Finally if $p=2 /(n+1)$, one can write $F=$ $p F^{(1)}+(1-p) F^{(2)}$ with

$$
F^{(1)}=\frac{1}{p}\left(\left(p-\frac{1-p}{n}\right) \delta_{0}+\frac{1-p}{n} \delta_{n}\right) \quad \text { and } \quad F^{(2)}=\frac{1}{n} \sum_{i=1}^{n-1} \delta_{i} .
$$

Again we have $\mu\left(F^{(1)}\right)=\mu\left(F^{(2)}\right)=\mu$. By symmetry $\mu_{(2 k+1)}\left(F^{(2)}\right)=0$ and by Lemma $4.1 \mu_{(2 k+1)}\left(F^{(1)}\right)>0$. Thus Lemma 2.3 yields $\mu_{(2 k+1)}(F)>0$.

Lemma 4.4. If $F$ is of the form

$$
F=\frac{p}{m+1} \sum_{i=0}^{m} \delta_{i}+\frac{1-p}{n} \sum_{i=m+1}^{m+n} \delta_{i}
$$


where $m$ is a non-negative integer, $n$ is a positive integer, and $(m+1) /(m+n+1)<p<1$, then $\mu_{(2 k+1)}(F)$ $>0$ for every positive integer $k$.

Proof. Induction on $m$. The case $m=0$ is Lemma 4.3. Fix $m>0$. If $(m+n) /(m+n+1)<p<1$, then the result follows from Lemma 4.2. If $p=(m+n) /(m+n+1)$, one can write $F=\pi F^{(1)}+(1-\pi) F^{(2)}$ with $\pi=p /(m+1)+(m+n)(1-p) / n$ and with

$$
F^{(1)}=\frac{1}{\pi}\left(\frac{p}{m+1} \delta_{0}+\frac{1-p}{n} \sum_{i=1}^{m+n} \delta_{i}\right) \quad \text { and } \quad F^{(2)}=\frac{1}{1-\pi}\left(\frac{p}{m+1}-\frac{1-p}{n}\right) \sum_{i=1}^{m} \delta_{i} .
$$

Again we have $\mu\left(F^{(1)}\right)=\mu\left(F^{(2)}\right)=\mu$. By symmetry $\mu_{(2 k+1)}\left(F^{(2)}\right)=0$ and by the induction hypothesis $\mu_{(2 k+1)}\left(F^{(1)}\right)>0$. Thus Lemma 2.3 yields $\mu_{(2 k+1)}(F)>0$. Finally if $(m+1) /(m+n+1)<p<(m+$ $n) /(m+n+1)$, one can write $F=\pi F^{(1)}+(1-\pi) F^{(2)}$ with $\pi=p /(m+1)+(m+n) b$, with

$$
F^{(1)}=\frac{1}{\pi}\left(\frac{p}{m+1} \delta_{0}+b \sum_{i=1}^{m+n} \delta_{i}\right)
$$

and

$$
F^{(2)}=\frac{1}{1-\pi}\left(\left(\frac{p}{m+1}-b\right) \sum_{i=1}^{m} \delta_{i}+\left(\frac{1-p}{n}-b\right) \sum_{i=m+1}^{m+n} \delta_{i}\right)
$$

and with $b$ such that $\mu\left(F^{(1)}\right)=\mu\left(F^{(2)}\right)=\mu$. The induction hypothesis yields $\mu_{(2 k+1)}\left(F^{(1)}\right)>0$ and $\mu_{(2 k+1)}\left(F^{(2)}\right)>0$. Thus Lemma 2.3 yields $\mu_{(2 k+1)}(F)>0$.

Recall that for $u, v \in L_{d}, U_{u, v}^{(d)}$ denotes the uniform distribution on $[u, v] \cap L_{d}$.

Theorem 4.5. Let $F$ be a probability measure of the form $F=p U_{s, t}^{(d)}+(1-p) U_{r, u}^{(d)}$ with $0<p<1$ and $r, s, t$, $u \in L_{d}$ such that $r \leqslant s \leqslant t<u$ and $\frac{1}{2}(s+t)<\frac{1}{2}(r+u)$. Then $\mu_{(2 k+1)}(F)>0$ for every positive integer $k$.

Proof. If $r<s$, a little algebra shows that $F$ can be written as

$$
F=p_{1}\left(p_{2} U_{r, s}^{(d)}+\left(1-p_{2}\right) U_{r, u}^{(d)}\right)+\left(1-p_{1}\right)\left(p_{3} U_{s, t}^{(d)}+\left(1-p_{3}\right) U_{s, u}^{(d)}\right)
$$

for some $p_{1}, p_{2}, p_{3} \in(0,1)$ such that $\mu(F)=\mu\left(p_{2} U_{r, s}^{(d)}+\left(1-p_{2}\right) U_{r, u}^{(d)}\right)=\mu\left(p_{3} U_{s, t}^{(d)}+\left(1-p_{3}\right) U_{s, u}^{(d)}\right)$. Thus, in view of Lemma 2.3, it suffices to consider the case $r=s$. First, consider the case $d=0$. If $s<t$, then after some elementary integration and algebra one can write the $(2 k+1)$ st central moment of $p U_{s, l}^{(d)}+(1$ $-p) U_{s, u}^{(d)}$ as

$$
\frac{(s \mu)^{2 k+2}}{2 k+2}\left\{\frac{p}{t-s}\left(\left(\frac{t-\mu}{s-\mu}\right)^{2 k+2}-1\right)+\frac{1-p}{u-s}\left(\left(\frac{u-\mu}{s-\mu}\right)^{2 k+2}-1\right)\right\}
$$

where $\mu=\frac{1}{2} p(s+t)+\frac{1}{2}(1-p)(s+u)$. The quantity $(s-\mu)^{2 k+2} /(2 k+2)$ is strictly positive for every positive integer $k$. Now consider the quantity inside the curl braces. It is equal to 0 if $k=0$, it is strictly positive if $k=1$, and it is convex in $k$, for $k \in\{0,1,2,3, \ldots\}$. Thus it is strictly positive for every positive integer $k$. The case $s=t$ can be treated similarly. Now consider the case $d>0$. Without any loss of generality one can assume that $d=1$ and $s=0$. In this case the desired result is just a restatement of Lemma 4.4.

\section{Acknowledgement}

I wish to thank Professor Dennis Gilliland for bringing to my attention the problem treated here. 


\section{References}

Birnbaum, Z.W. (1950), Effect of linear truncation on a multinormal population, Ann. Math. Statist. 21, 272-279.

Frame, J.S. and D.C. Gilliland, (1985), Votes and a half-binomial, Discrete Appl. Math. 12, 31-40.

Gilliland, D.C. and T. Hsing (1988), Private communication.

Gill, R. and D.C. Gilliland (1989), Problem 234, Statist. Neerlandica 43, 66.

Hannan, J. and E.J.G. Pitman (1965), Symmetric unimodal variables conditioned to upper tails have positive central moments, Tech. Rept., Dept. of Probab. and Statist., Michigan State Univ. (East Lansing, MI).

MacGillivray, H.L. (1981), The mean, median, mode inequality and skewness for a class of densities, Austral. J. Statist. 23, 247-250.

Runnenburg, J.Th. (1978), Mean, median, mode, Statist. Neerlandica 32, 73 -79. 